\title{
Detecting Congestive Heart Rate Variability Failure Using Pointcaré Trend Analysis Plot
}

\author{
Hoang Chu Duc, Hung Pham Manh, Thuan Nguyen Duc, and Dung Nguyen Viet
}

\begin{abstract}
The function of heart rate variability (HRV) has been studied over the years, but less is known about the factors predicting recovery from work stress during sleep. The availability to register reliable data for short-time HRV has raised the interest to find the congestive HRV signal. The objective of this study was to identify individual-level factors related to work and leisure-time predicting the recovery of autonomic nervous system (ANS) during congestion measured by 24-h HRV. 15 subjects (11 men, aged 22 to 71 , and 4 women, aged 54 to 63) with severe congestive heart failure (NYHA class 3-4). This group of subjects was part of a larger study group receiving conventional medical therapy prior to receiving the oral inotropic agent, milrinone. Questionnaire data was gathered with a self-administered questionnaire of individual characteristics, perceived work ability, stress and psychological resources. The individual recordings are each about 20 hours in duration, and contain two ECG signals each sampled at 250 samples per second with 12-bit resolution over a range of \pm 10 millivolts. In this study, we only calculate first 15 minus of each subject.
\end{abstract}

Index Terms-Pointcaré plot, heart rate variability, chaos, congestive heart failure.

\section{INTRODUCTION}

Heart failure (HF), often called congestive heart failure (CHF) or congestive cardiac failure (CCF), occurs when the heart is unable to provide sufficient pump action to maintain blood flow to meet the needs of the body [1]-[3]. Heart failure can cause a number of symptoms including shortness of breath, leg swelling, and exercise intolerance. The condition is diagnosed by patient physical examination and confirmed with echocardiography. Blood tests help to the etiology diagnosis. Treatment depends from severity and etiology of heart failure. In a chronic patient already in a stable situation, treatment commonly consists of lifestyle measures such as smoking cessation, light exercise, dietary changes, and medications. Sometimes, depending from etiology, it is treated with implanted devices (pacemakers or ventricular assist devices) and occasionally a heart transplant is required.

Common causes of heart failure include myocardial infarction and other forms of ischemic heart disease,

Manuscript received April 9, 2013; revised July 11, 2013.

Hoang Chu Duc, Thuan Nguyen Duc, and Hung Pham Manh are with the Department of Electronics Technology and Biomedical Engineering, School of Electronics and Telecommunications, Hanoi University of Scicence and Technology, Hanoi, Vietnam (e-mail: hoang.chuduc@hust.edu.vn, thuan.nguyenduc@hust.edu.vn).

Dung Nguyen Viet is with Center of Electronics and Biomedical, Hanoi University of Scicence and Technology, Hanoi, Vietnam (e-mail: trung.laihuuphuong@hust.edu.vn). hypertension, valvular heart disease, and cardiomyopathy [4]. The term heart failure is sometimes incorrectly used for other cardiac-related illnesses, such as myocardial infarction (heart attack) or cardiac arrest, which can cause heart failure but are not equivalent to heart failure.

Heart failure is a common, costly, disabling, and potentially deadly condition. [4] In developed countries, around $2 \%$ of adults suffer from heart failure, but in those over the age of 65 , this increases to $6-10 \%$ [4], [5]. It is widely recognized that exercise training induces acute and chronic adaptations in heart rate (HR), but the exact mechanisms that mediate these changes are not clear [1]-[4]. It is hypothesized that training can affect autonomic regulation causing reduction in the sympathetic nerve activity and increase in the parasympathetic outflow [5], [6]. Previous studies have shown that the autonomic modulation of HR can be studied by non-invasive methods utilizing heart rate variability (HRV) [7]-[12]. The HRV is associated with sympathovagal balance and it can be a practical and accurate method to assess the effects of acute exercise and training on the autonomic modulation of HR [6]-[13]. It is derived from analysis of consecutive beat-to-beat oscilations of sinus rhythm in time or frequency domains, which are mainly mediated by the autonomic nervous system branches' activities. However, other neural, humoral, and metabolic factors might also induce changes on HR and on HRV parameters. Another study investigated the effects of aerobic training on HRV response during a progressive cycle ergometer test. The training involved cycling during $30 \mathrm{~min}$ at $50 \%$ of the difference between peak work rate during the progressive test and HRV threshold. The sessions were performed three times per week throughout three weeks. The results showed that moderate-intensity training caused an increase in work rate at HRV threshold while no significant changes were observed in the control group. However, during progressive exercise test, the effects of high-intensity interval training on HRV response have not been established yet.

Therefore, the purpose of this study was to investigate the effects of high-intensity interval training on HRV threshold and on HRV-work rate curve during progressive exercise. We have hypothesized that significant changes would occur in the autonomic cardiac control in response to this form of training and consequently, HRV-work rate curve during progressive exercise would be shifted to the upward and to the right directions, with concomitant reduction in heart rate in submaximal stages.

\section{Methods}

\section{A. Data Acquisitions}

This database includes long-term ECG recordings from 15 
subjects (11 men, aged 22 to 71 , and 4 women, aged 54 to 63 ) with severe congestive heart failure (NYHA class 3-4). This group of subjects was part of a larger study group receiving conventional medical therapy prior to receiving the oral inotropic agent, milrinone. Further details about the larger study group are available in the first reference cited above. A number of additional studies have made use of these recordings; see the additional references below.

The individual recordings are each about 20 hours in duration, and contain two ECG signals each sampled at 250 samples per second with 12-bit resolution over a range of \pm 10 millivolts. In this study, we only calculate first 15 minus of each subject. The original analog recordings were made at Boston's Beth Israel Hospital (now the Beth Israel Deaconess Medical Center) using ambulatory ECG recorders with a typical recording bandwidth of approximately $0.1 \mathrm{~Hz}$ to 40 $\mathrm{Hz}$. Annotation files (with the suffix .ecg) were prepared using an automated detector and have not been corrected manually.

\section{B. Poincaré Plot Method}

Standard HR and its variability analysis have been performed in parallel to Poincare plots of RR intervals during every testing condition and overall Poincare plot. Minimal and maximal HR frequency, the difference between of them $(\triangle \mathrm{RRr})$ and total $H R$ variability $(\Delta \mathrm{RRt})$ as a measure of reflex and tonic HR control, correspondingly, were measured. Correlation between of standard and new parameters was analysed.

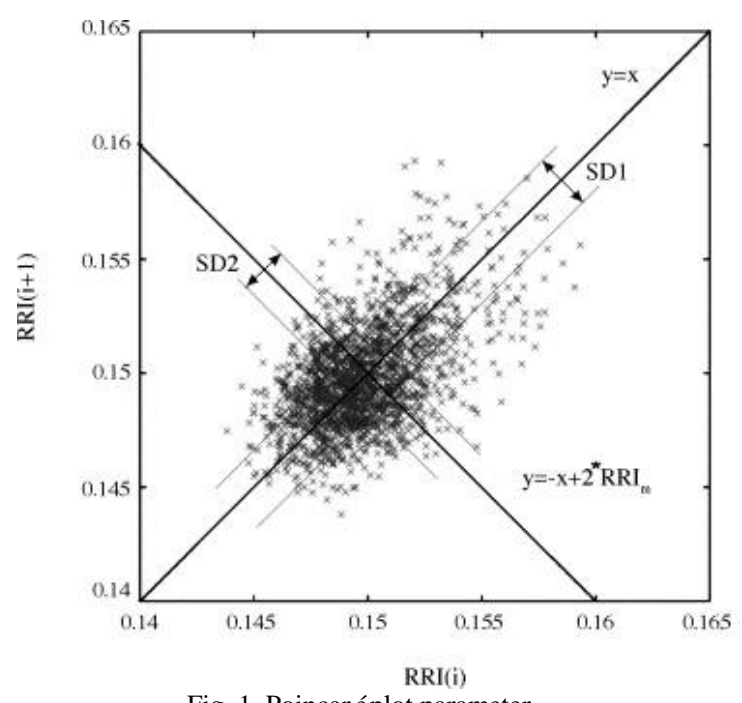

Fig. 1. Poincaré plot parameter.

The Poincaré plot is a scatterplot of the current R-R interval plotted against the preceding R-R interval. Poincaré plot analysis is a quantitative visual technique, whereby the shape of the plot is categorized into functional classes. The plot provides summary information as well as detailed beat-to-beat information on the behavior of the heart. Points above the line of identity indicate R-R intervals that are longer than the preceding R-R interval, and points below the line of identity indicate a shorter R-R interval than the previous. Accordingly, the dispersion of points perpendicular to the line of identity (the "width") reflects the level of short-term variability. This dispersion can be quantified by the standard deviation of the distances the points lie from the line of identity. This measure is equivalent to the standard deviation of the successive differences of the R-R intervals [standard deviation of successive differences (SDSD) or root-mean-square of successive differences (RMSSD)] [14]. The standard deviation of points along the line of identity (the "length") reflects the standard deviation of the R-R intervals (SDRR). Fig. 1 details these quantitative measures of Poincaré plot shape.

Poincaré plots appear under different names in the literature: scatter plots, first return maps, and Lorenz plots being prominent terms. A distinct advantage of Poincaré plots is their ability to identify beat-to-beat cycles and patterns in data that are difficult to identify with spectral analysis [15], [16].

Considering the complex control systems of the heart it is reasonable to assume that nonlinear mechanisms are involved in the genesis of HRV.

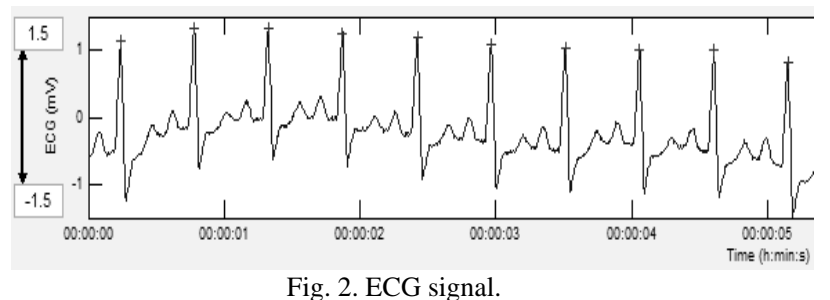

The nonlinear properties of HRV have been analyzed using measures such as Poincaré plot [17], [18], approximate and sample entropy [19], [20], detrended fluctuation analysis [21], [22], correlation dimension, and recurrence plots. During the last years, the number of studies utilizing such methods have increased substantially. The downside of these methods is still, however, the difficulty of physiological interpretation of the results.

One commonly used nonlinear method that is simple to interpret is the so-called Poincare plot. It is a graphical representation of the correlation between successive $R R$ intervals, i.e. plot of $R R j+1$ as a function of $R R j$.

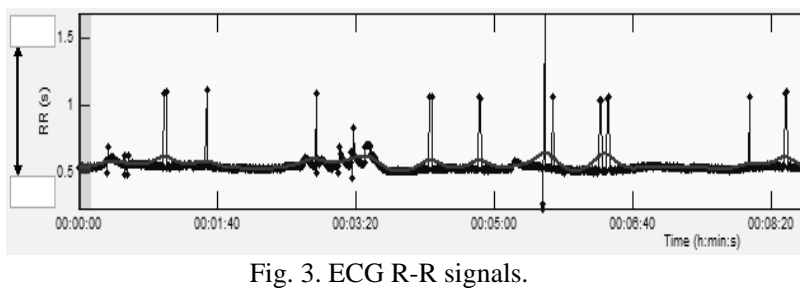

The shape of the plot is the essential feature. A common approach to parameterize the shape is to fit an ellipse to the plot as shown in Fig. 1. The ellipse is oriented according to the line-of-identity $(R R j=R R j+1)$ [17]. The standard deviation of the point's perpendicular to the line-of-identity denoted by SD1 describes short-term variability which is mainly caused by RSA. It can be shown that SD1 is related to the time-domain measure SDSD according to [17].

$$
S D 1^{2}=\frac{1}{2} S D S D^{2}
$$

The standard deviation along the line-of-identity denoted by SD2, on the other hand, describes long-term variability and has been shown to be related to time-domain measures SDNN and SDSD by [17]. 


$$
S D 2^{2}=2 S D N N^{2}-\frac{1}{2} S D S D^{2}
$$

The instantaneous beat-to-beat variability of the data was derived from SD1 index. Details of SD1 analysis were described previously. The SD1 index was plotted against work rate and the first intensity at which the SD1 index reached values equal to or lower than $3 \mathrm{~ms}$ was defined as the HRV threshold. The mean HR of each stage was also calculated and plotted against work rate to estimate the HR at HRV threshold. The maximal work rate and maximal HR computed during the incremental tests were also compared in the pre- and post-training.

\section{RESULTS}

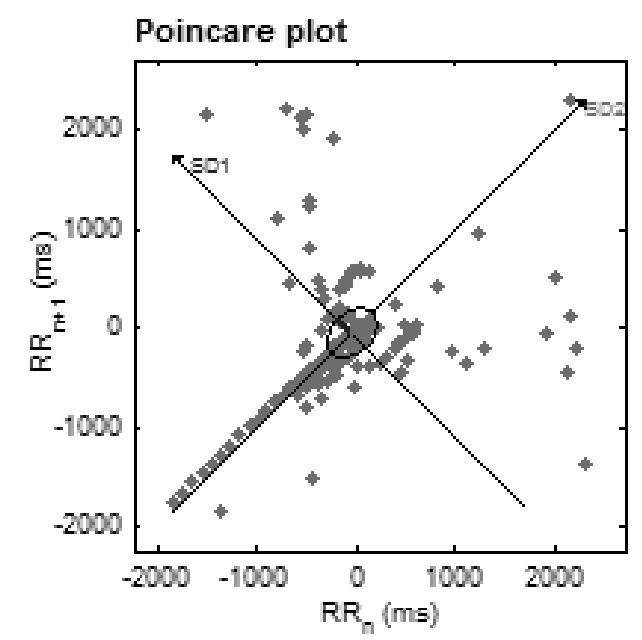

Fig. 4. Poincaré plot of a subject. Length and width are shown graphically on plot.

TABLE I: THE POINCARÉ RESUltS OF 15 SUBJECT

\begin{tabular}{llll}
\hline \hline Subjects & $\begin{array}{c}\text { Sample } \\
\text { Time } \\
\text { (seconds) }\end{array}$ & \multicolumn{1}{c}{$\begin{array}{c}\text { Poincare plot } \\
\text { SD1(mm) }\end{array}$} & $\begin{array}{c}\text { Poincare plot } \\
\text { SD1(mm) }\end{array}$ \\
\hline 1 & Channel 1 & Channel 2 & 99.646 \\
\hline 2 & 900 & 102.35 & 53.029 \\
\hline 3 & 900 & 62.185 & 92.441 \\
\hline 4 & 900 & 95.96 & 92.023 \\
\hline 5 & 900 & 83.982 & 73.199 \\
\hline 6 & 900 & 62.031 & 65.465 \\
\hline 7 & 900 & 92.301 & 63.796 \\
\hline 8 & 900 & 87.857 & 53.033 \\
\hline 10 & 900 & 56.268 & 42.171 \\
\hline 11 & 900 & 48.473 & 21.89 \\
\hline 12 & 900 & 22.479 & 44.577 \\
\hline 13 & 900 & 44.56 & 332.71 \\
\hline 14 & 900 & 162.28 & 160.3 \\
\hline 15 & 900 & 35.668 & 289.16 \\
\hline \hline
\end{tabular}

Based on a review of the state of the art of software related to the HRT analysis, we motivate the development of this open source software platform which could be an interesting tool both for studying HRT or performing clinical experiments for research purposes. We present the results of the application of our package to two ECG databases from Physionet of patients suffering from Chronic Heart Failure. Values for average work rate performed during each week are presented in Fig. 3

We performed a discriminant analysis using the HRT parameters in order to distinguish between severe CHF from mild CHF. We adopted as discriminant function the Mahalanobis distances with stratified covariance estimates. The computed HRT parameters enables severe CHF patients to be distinguished from mild $\mathrm{CHF}$ ones with a sensitivity and a specificity rate as see on Table I.

\section{DISCUSSIONS}

When we analyzed the RR interval time series of healthy subjects with the Poincaré plot method in the congestive phase, there are no oscillations, but when we applied this method to the series of the CHF patients we observe oscillations.

In the Poincaré plot we have showed that these oscillations are associated with periodicities in the time series, we think this fact is very important because we did not find these oscillations in healthy persons in neither of the two phases, in the CHF patients they are encountered in the sleep phase of many patients and hardly ever in the wake phase.

Our results show that this appearance of periodic components can be observed in the graph that is used to calculate the Poincaré fractal dimension. This can be shown generating a time series of known fractal dimension, after this we add this time series several periodic components and upon calculating the Higuchi's graph we observe oscillations, we can calculate the frequencies of these oscillations and they correspond precisely to the periodic components that we added.

The aim of this study is to clarify the basic relationship between measurements of HRV and severe congestive heart failure. The most rapid increase in sympathetic effect and decrease in vagal-tone in the early morning hours in normal individual are related to cardiovascular events.

\section{CONCLUSIONS}

As proved by the results of this research, HRT measurements appear to be an effective instrument for Congestive Heart Failure severity assessment. In particular we found that the combined use of two of the HRT parameters computed through our Matlab tool, increases the discrimination performances, especially in terms of sensitivity and accuracy. We firmly believe that the results of our study and of previously published papers about HRT encourage further investigations concerning HRT parameters in many other clinical situations and, in this scenario, the developed Matlab tools could show its potentiality for future researches.

\section{REFERENCES}

[1] J. Wahlund, "Determination of physical working capacity," Acta Med Scand Suppl., 1948, vol. 215, pp. 1-78.

[2] P.-O. Åstrand and B. Saltin, "Maximal oxygen uptake and heart rate in various types of muscular activity," J Appl Physiol, 1961, vol. 16, pp. 977-981.

[3] B. Ekblom, P. O. Astrand, B. Saltin, J. Stenberg, and B. Wallstrom, "Effect of training on circulatory response to exercise," J Appl Physiol, 1968, vol. 24, pp. 518-528.

[4] D. Linnarson, "Dynamics of pulmonary gas exchange and heart rate changes at start and end of exercise," Acta Physiol Scand, 1974, vol. 415 , pp. 1-68. 
[5] J. B. Carter, E. W. Banister, and A. P. Blader, "The effect of age and gender on heart rate variability after endurance training," Med Sci Sport Exer., 2003, vol. 35, no. 8, pp. $1333-1340$.

[6] M. P. Tulppo, A. J. Hautala, T. H. Mäkikallio, R. T. Laukkanen, S. Nissilä, R. L. Hughson, and H. V. Huikuri, "Effects of aerobic training on heart rate dynamics in sedentary subjects," J Appl Physiol., 2003, vol. 95 , pp. $364-372$.

[7] A. Malliani, M. Pagani, F. Lombardi, and S. Cerutti, "Cardiovascular neural regulation explored in the frequency domain," Circulation, 1991, vol. 84, pp. 482-492.

[8] M. Bootsma, C. A. Swenne, H. H. V. Bolhuis, P. C. Chang, V. M. Cats, and A. V. Bruschke, "Heart rate and heart rate variability as indexes of sympathovagal balance,” Am J Physiol (Heart Circ. Physiol.), 1994, vol. 266, pp. H1565-H1571.

[9] Task Force of the European Society of Cardiology and the North American Society of Pacing and Electrophysiology: Heart rate variability, "Standards of measurement, physiological interpretation, and clinical use," Circulation, 1996, vol. 93, pp. 1043-1065.

[10] H. M. Stauss, "Heart rate variability," American Journal of Physiology-Regulatory, Integrative and Comparative Physiology, 285, pp. R927-R931, 2003.

[11] A. Hautala, "Effect of physical exercise on autonomic regulation of heart rate," Academic Dissertation (Faculty of Medicine) - University of Oulu, Finland, 2004, pp. 76.

[12] L. Fronchetti, F. Y. Nakamura, C. A. Aguiar, and F. R. De-Oliveira, "Indicadores de regulação autonômica cardíaca em repouso e durante exercício progressivo - Aplicação do limiar de variabilidade de freqüência cardíaca," Rev Port Cien Desporto, vol. 6, no. 1, pp. 21-28, 2006.

[13] L. Mourot, M. Bouhaddi, S. Perrey, J. D. Rouillon, and J. Regnard, "Quantitative Poincaré plot analysis of heart rate variability: effect of endurance training," Eur J Appl Physiol, vol. 91, pp. 79-87, 2004.

[14] P. W. Kamen and A. M. Tonkin, "Application of the Poincaré plot to heart rate variability: a new measure of functional status in heart failure," NZ J Med., Aust. 1995, vol. 25, pp. 18-26.

[15] M. A. Woo, W. G. Stevenson, D. K. Moser, and H. R. Middlekauff, "Complex heart rate variability and serum norepinephrine levels in patients with advanced heart failure," J Am Coll Cardiol., vol. 23, pp. 565-591, 1994.

[16] M. A. Woo, W. G. Stevenson, D. K. Moser, R. B. Trelease, and R. H. Harper, "Patterns of beat-to-beat heart rate variability in advanced heart failure," Am Heart J., vol. 123, pp. 704-710, 1992.

[17] M. Brennan, M. Palaniswami, and P. Kamen, "Do existing measures of Poincare plot geometry reect nonlinear features of heart rate variability," IEEE Trans Biomed Eng., vol. 48, no. 11, pp. 1342-1347, November 2001.

[18] S. Carrasco, M. J. Caitan, R. Gonzalez, and O. Yanez, "Correlation among Poincare plot indexes and time and frequency domain measures of heart rate variability," J Med Eng Technol., vol. 25, no. 6, pp. 240-248, November/December 2001.

[19] J. A. Richman and J. R. Moorman, "Physiological time-series analysis using approximate entropy and sample entropy," Am J Physiol., vol. 278, pp. H2039-H2049, 2000.
[20] Y. Fusheng, H. Bo, and T. Qingyu, "Approximate entropy and its application in biosignal analysis," in Nonlinear Biomedical Signal Processing: Dynamic Analysis and Modeling, M. Akay, ed., vol. II, chapter 3, pp. 72-91, New York: IEEE Press, 2001.

[21] C.-K. Peng, S. Havlin, H. E. Stanley, and A. L. Goldberger "Quantication of scaling exponents and crossover phenomena in nonstationary heartbeat time series," Chaos, vol. 5, pp. 82-87, 1995.

[22] T. Penzel, J. W. Kantelhardt, L. Grote, J.-H. Peter, and A. Bunde, "Comparison of detrended fluctuation analysis and spectral analysis for heart rate variability in sleep and sleep apnea," presented at the IEEE Trans Biomed Eng, vol. 50, no. 10, pp. 1143-1151, October 2003.

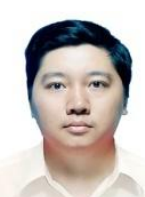

Hoang Chu Duc is a researcher at Department of Electronics and Biomedical Engineering, School of Electronics and Telecommunication, Hanoi University of Science and Technology.

He has 8 years' experience at researcher in biomedical equipment design, signal processing and teaching. His key qualifications (relevant to the project): Analysis, design, implementation of information systems and management for hospitals and clinics; Quality management for software processing; Coordinating projects and planning medical equipment; Supervision for IT projects; Preparing bidding documents, bid evaluation; Project management and coordination for such domestic agencies as FPT, Viettel, Panasonic, Department of IT, Ministry of Health, CMC, IBM, Oracle, hospitals as well as foreign partners from Singapore, Japan, Australia, Malaysia. Since 2010, he does PhD research with project "Impact analysis between the ECG signal and the common defense of the Vietnamese"

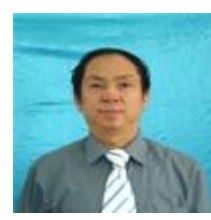

Thuan Nguyen Duc is a lecture at Department of Electronics and Biomedical Engineering, School of Electronics and Telecommunication, Hanoi University of Science and Technology. He has over 30 years' experience in biomedical equipment design, signal processing and teaching.

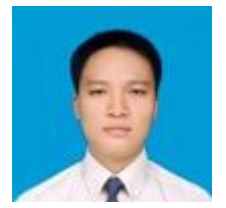

Hung Pham Manh is a researcher at Department of Electronics and Biomedical Engineering, School of Electronics and Telecommunication, Hanoi University of Science and Technology. He has over 7 years' experience in biomedical equipment design, signal processing and teaching.

Dung Nguyen Viet is a director at Center of Electronics and Biomedical, Hanoi University of Scicence and Technology, Hanoi. He has over 10 years' experience in biomedical equipment design, signal processing and teaching. 